LIVER

\title{
Fatigue and primary biliary cirrhosis: association of globus pallidus magnetisation transfer ratio measurements with fatigue severity and blood manganese levels
}

\author{
D M Forton, N Patel, M Prince, A Oatridge, G Hamilton, J Goldblatt, J M Allsop, J V Hajnal, \\ H C Thomas, M Bassendine, D E J Jones, S D Taylor-Robinson
}

Gut 2004;53:587-592. doi: 10.1136/gut.2003.016766 See end of article for
authors' affiliations
......................
Correspondence to:
Dr D Forton, Hepatology
Section, Faculty of
Medicine, Imperial College
London, 1Oth floor QEQM
Building, St Mary's
Hospital, South Wharf Rd,
London W2 1NY, UK;
d.forton@imperial.ac.uk
Accepted for publication
25 June 2003

\begin{abstract}
Background and aim: Fatigue is the commonest symptom in primary biliary cirrhosis (PBC), affecting individuals at all stages of disease. The pathogenesis of fatigue in PBC is unknown although rat models suggest a central nervous system (CNS) cause. We examined the hypothesis that a CNS abnormality related to cholestasis, rather than cirrhosis per se, underlies this symptom.

Patients and methods: Fourteen patients with precirrhotic PBC (stage I-II disease), four patients with stage III-IV PBC, and 11 healthy women were studied using cerebral magnetisation contrast imaging and proton magnetic resonance spectroscopy (MRS).

Results: The globus pallidus magnetisation transfer ratio (MTR), a quantifiable tissue characteristic that may be abnormal in the presence of normal magnetic resonance imaging, was significantly reduced in precirrhotic PBC patients compared with healthy controls. These measurements correlated with blood manganese levels and were more abnormal in the more fatigued subjects. There were no differences in MRS measurements between the three study groups, suggesting that the abnormal MTR was not related to hepatic encephalopathy.

Conclusion: This study suggests that impairments in liver function in PBC may adversely affect the brain long before the development of cirrhosis and hepatic encephalopathy, possibly as a result of altered manganese homeostasis within the CNS.
\end{abstract}

$\mathrm{F}$ atigue is a common, yet highly subjective, complaint among patients with primary biliary cirrhosis (PBC). ${ }^{1}$ With the advent of screening liver function tests and serological testing, the diagnosis of PBC is usually made early in the course of the natural history of the condition. ${ }^{2}$ Consequently, early presenting symptoms such as fatigue have assumed greater importance. The prevalence of fatigue in $\mathrm{PBC}$ has been reported to be as high as $80 \%^{3-5}$ although these clinic based studies may have been subject to selection bias. A recent geographically based survey, using a well validated fatigue questionnaire, reported significant fatigue in $40 \%$ of PBC patients. ${ }^{6}$ Fatigue is commonly of sufficient severity to significantly impair health related quality of life and, importantly, affects individuals at all stages of disease. ${ }^{5}$

Despite the prevalence of fatigue in $\mathrm{PBC}$, the current understanding of its aetiology is limited. Although tools have been developed to measure fatigue in $\mathrm{PBC}^{7}$ very few clinical studies have addressed its pathophysiology. The symptom complex suggests a central nervous system (CNS) mechanism although there are conflicting reports regarding the presence of peripheral neuromuscular impairment. ${ }^{8}$ Studies on bile duct resected rats, using animal models of fatigue assessment, have suggested three possible mechanisms: (1) decreased hypothalamic release of corticotrophin releasing hormone $^{10}$; (2) abnormal serotonergic neurotransmission ${ }^{11}$; and (3) increased brain sensitivity to interleukin $1 \beta{ }^{12}$ However, whether these mechanisms are important in humans and how they are related to chronic cholestatic liver disease remains unclear.

We considered that impaired biliary excretion might result in accumulation of substances within the brain, including heavy metals such as manganese which are normally excreted via the biliary route. There is post-mortem evidence for manganese accumulation within the globus pallidus in patients dying with established cirrhosis. ${ }^{13}{ }^{14}$ Furthermore, marked hyperintensity of the globus pallidus (GP) on $T_{1}$ weighted magnetic resonance imaging (MRI) has been reported both in patients with cirrhosis ${ }^{13}{ }^{15-19}$ and in patients with hypermanganesaemia due to occupational exposure, ${ }^{20}$ biliary atresia, ${ }^{21}$ and total parenteral nutrition..$^{22}$

We hypothesised that the fatigue experienced by patients with PBC, in the absence of cirrhosis, may occur as a consequence of increased GP manganese concentrations as a result of impaired biliary excretion. We used a combination of MRI and MR spectroscopy (MRS) to investigate this.

\section{METHODS}

\section{Patient groups}

Fourteen women (mean age 60 years (range 41-76)) with a definite diagnosis of $\mathrm{PBC}$, based on an antimitochondrial antibody at a titre of $\geqslant 1: 40$ by immunofluorescence and diagnostic liver histology were studied. All had stage I-II disease based on histological assessment of biopsies taken within the preceding year. Four additional women with stage III or IV disease (mean age 48 years (range 39-59)) were also studied. Mean (SD) serum bilirubin and albumin levels were 7.6 (2.5) $\mu \mathrm{mol} / \mathrm{l}$ and $39.4(2.4) \mathrm{g} / \mathrm{l}$, respectively, in stage I-II patients and 18.8 (12.5) $\mu \mathrm{mol} / \mathrm{l}$ and 38.0 (2.9) g/l in stage IIIIV patients.

Abbreviations: $\mathrm{PBC}$, primary biliary cirrhosis; CNS, central nervous system; MRI, magnetic resonance imaging; MRS, magnetic resonance spectroscopy; MTR, magnetisation transfer ratio; GP, globus pallidus; $\mathrm{ROI}$, region of interest; PU, putamen; WM, white matter 
Eleven healthy women (mean age 47 years (range 38-65)) were recruited from hospital staff to provide control data for MRI and MRS.

Exclusion criteria for the study were a history of any additional liver disease, CNS altering medication, nocturnal pruritus, claustrophobia, and the presence of ferromagnetic prostheses or cardiac pacemakers

\section{Patient assessment}

All patients completed the fatigue impact scale, a measure of the impact of fatigue on daily functioning, comprising physical, cognitive, and psychosocial elements, on the day they were studied. These assessment tools have previously been validated for use in PBC. ${ }^{67}$ All patients gave blood for measurement of manganese concentrations, as well as other metals (copper, iron, mercury, and cadmium).

\section{Ethics}

Ethics approval was obtained from the ethics committees of the Imperial College School of Medicine (Rec 4047/93) and was in accordance with the 1975 Helsinki Declaration on Human Rights. All subjects provided written informed consent.

\section{Magnetic resonance}

All subjects were studied with a protocol that included combined cerebral MRI and proton $\left({ }^{1} \mathrm{H}\right)$ MRS.

\section{Magnetic resonance imaging}

We anticipated that conventional $\mathrm{T}_{1}$ weighted imaging might not detect small changes in $T_{1}$ shortening in this cohort of patients with early liver disease due to PBC. We therefore employed an MR sequence to measure magnetisation transfer ratios (MTRs). The MTR is a quantitative tissue characteristic that reflects the behaviour of normally MR invisible protons bound to macromolecules. ${ }^{23}$ It can be considered to give enhanced image contrast and may detect brain parenchymal changes that may not be visible using standard MR techniques. Patients were scanned using a birdcage headcoil in a 1.5 Tesla Eclipse MR scanner (Philips Medical Systems Inc., Cleveland, Ohio, USA). An initial spin echo proton density sequence (TR/TE 2000/20 ms) was followed by the MT sequence (TR/TE 2000/20 ms) with an off resonance radiofrequency $(\mathrm{RF})$ pulse (frequency offset $=1000 \mathrm{~Hz}$, peak amplitude $=10 \mu \mathrm{T}$, duty cycle $=22 \%$ ). These values were selected to optimise the MT effect within energy constraints.

Regions of interest (ROIs) were selected bilaterally in the $\mathrm{GP}$, head of caudate, putamen (PU), thalamus, and frontal white matter (WM). Signal intensity measures were obtained from ROIs in both hemispheres and a mean value was calculated. The MTR was calculated using the following formula:

$$
\mathrm{MTR}=1-\mathrm{SI}_{\mathrm{RF}} / \mathrm{SI}_{0}
$$

where $\mathrm{SI}_{\mathrm{RF}}$ is the signal intensity in the image employing the off resonance RF pulse and $\mathrm{SI}_{0}$ is the signal intensity in the initial proton density image. In order to control for interexamination system variability, MTR results were expressed as indices normalised to the frontal white matter $(\mathrm{ROI}-\mathrm{WM}) /(\mathrm{ROI}+\mathrm{WM})$ and putamen $(\mathrm{ROI}-\mathrm{PU}) /(\mathrm{ROI}+\mathrm{PU})$ in each patient.

\footnotetext{
${ }^{1} \mathrm{H}$ Magnetic resonance spectroscopy

${ }^{1} \mathrm{H}$ MRS is a technique that generates metabolic information and has been used by our group and others to study both the pathophysiology of hepatic encephalopathy and the neuropsychological symptoms of chronic hepatitis C infection. ${ }^{24-29}$
}

We utilised ${ }^{1} \mathrm{H}$ MRS in this study to exclude the presence of hepatic encephalopathy and to investigate whether there are any fatigue associated ${ }^{1} \mathrm{H}$ MRS features in patients with PBC that may mirror our findings in patients with precirrhotic hepatitis C infection. Two $8 \mathrm{~cm}^{3}$ sized voxels were positioned in the basal ganglia and in the white matter at the level of the centrum semiovale. Single voxel ${ }^{1} \mathrm{H}$ MRS examinations were performed using an automated PRESS sequence (TR $1500 \mathrm{~ms}$, TE $135 \mathrm{~ms}$, and 128 acquisitions). ${ }^{28}$ In each case, MR spectra were analysed by a single observer who was blinded to the clinical status of the patients. Peak areas were measured for choline, creatine, and $N$-acetylaspartate ${ }^{30}$ using Philips proprietary software (Philips Medical Systems Inc.). Peak area ratios for choline/creatine were then calculated.

\section{Statistical methods}

Data were checked for normality using the Shapiro-Wilk test. Between group comparisons were made with the Student's $t$ test or the Mann-Whitney U test, as appropriate. Correlations were tested with Pearson's correlation. When multiple correlations were tested, a correction factor of four, corresponding to the number of subcortical ROIs, was used. All tests were two tailed. Statistical analyses were performed using SPSS version 10 (SPSS Inc., North Carolina, USA).

\section{RESULTS}

Fatigue

Individual total fatigue impact scores are shown in fig 1: 50\% of patients had high total fatigue scores $(>67)$, as defined in a recent geographically based survey from our group, ${ }^{6}$ and constituted the high fatigue group. In the stage III-IV group, two patients with cirrhosis had fatigue scores $>67$ whereas two stage III patients had low fatigue scores $(<25)$.

\section{Blood manganese concentrations}

Individual whole blood manganese and copper concentrations are shown in fig 2. Blood manganese and copper concentrations were elevated above the normal range in $5 / 18$ and $8 / 18$ patients, respectively. Other metal concentrations fell within the normal range. There were significant correlations between blood manganese and copper concentrations and the total fatigue impact score $(\mathrm{Mn}, r=0.43, \mathrm{p}=0.04$; $\mathrm{Cu}$, $r=0.48, \mathrm{p}=0.02$ ). In order to control for a possible effect of minimal hepatic encephalopathy, we excluded patients with stage III-IV PBC from the analysis. The mean blood

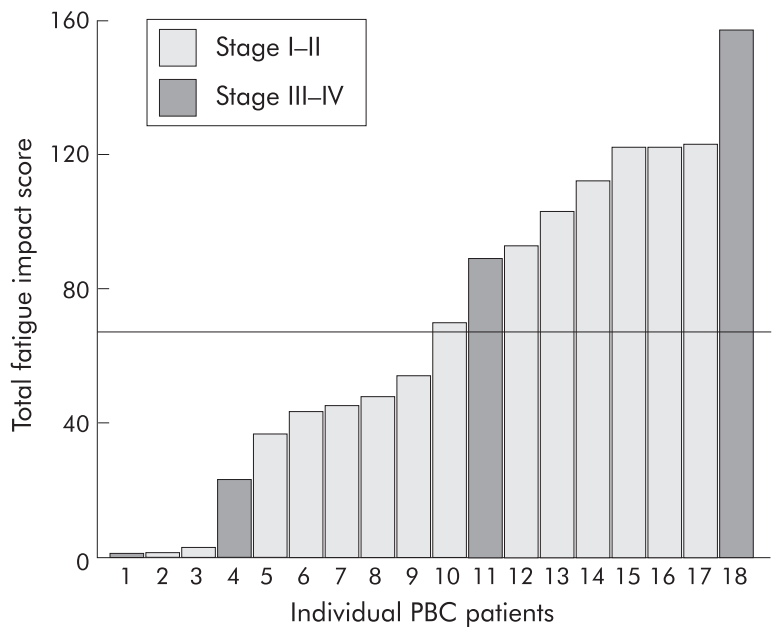

Figure 1 Total fatigue impact scores in patients with primary biliary cirrhosis (PBC). Patients were divided into high fatigue $(>67)$ and low fatigue $(<67)$ groups. 


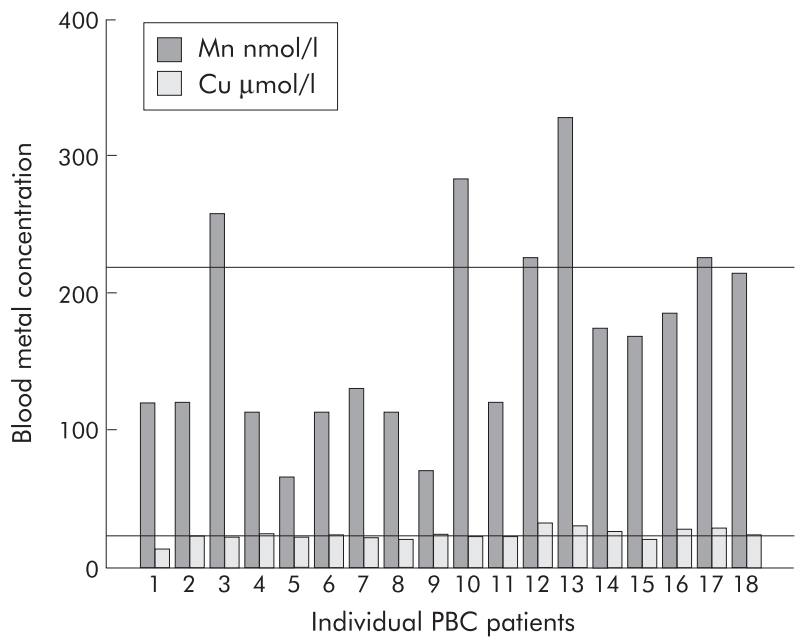

Figure 2 Whole blood manganese ( $\mathrm{Mn}$ ) and copper (Cu) concentrations in patients with primary biliary cirrhosis (PBC). Reference lines indicate the upper limit of normal for manganese $(218.5 \mathrm{nmol} / \mathrm{I})$ and copper $(22.9 \mu \mathrm{mol} / \mathrm{l})$.

manganese concentration was significantly higher in stage III patients with high fatigue levels than those with less fatigue (high fatigue 227.6 (59.6) nmol/l; low fatigue 124.7 (64.2) nmol/l; $\mathrm{p}=0.01$ ). There was no significant difference in mean blood copper concentrations between the high and low fatigue groups (high fatigue 26.6 (4.5) $\mu \mathrm{mol} / \mathrm{l}$; low fatigue 22.7 (1.5) $\mu \mathrm{mol} / \mathrm{l}$; NS).

\section{Magnetic resonance imaging}

Mean GP MTR in stage I-II PBC patients was significantly reduced compared with healthy controls (table 1). This reduction was restricted to the GP and was not seen in other ROIs when secondary comparisons were made (table 1). Data were also expressed as indices normalised to the WM and PU to control for inter-examination system variation. When normalised to the WM, the GP index was significantly reduced in stage I-II PBC patients (mean (SD) stage I-II PBC -0.074 (0.019); healthy controls -0.056 (0.019); $p=0.03$ ) (fig 3). Again, this pattern was restricted to the GP. The four stage III-IV patients had a greater reduction in the GP/WM index although this did not reach statistical significance (fig 3). There were no statistically significant differences when MTRs were normalised to the PU. We then examined whether there was a relationship between fatigue and the GP MTR indices. In stage I-II patients, the GP/WM and GP/PU MTR indices were significantly reduced in the high fatigue group compared with the low fatigue group (GP/WM high fatigue -0.084 (0.018); low fatigue -0.064 (0.014); $\mathrm{p}=0.04$ : GP/PU high fatigue 0.029 (0.021); low fatigue 0.055 (0.015); $\mathrm{p}=0.02$ ) (fig 4 ). There was a strong and significant negative

Table 1 Magnetic transfer ratios from the five regions of interest in the study groups

\begin{tabular}{lll}
\hline & Healthy controls $(\mathbf{n}=11)$ & PBC stage $\mathrm{I}-\mathrm{II}(\mathbf{n}=14)$ \\
\hline Globus pallidus & $35.05(1.83)$ & $33.24(2.02)^{*}$ \\
Thalamus & $34.70(1.61)$ & $34.24(2.04)$ \\
Putamen & $31.59(2.19)$ & $30.53(1.44)$ \\
Head of caudate & $31.23(1.50)$ & $30.55(1.57)$ \\
White matter & $39.19(1.32)$ & $38.51(1.44)$ \\
\hline
\end{tabular}

$\mathrm{PBC}$, primary biliary cirrhosis

${ }^{*} p<0.03$ compared with healthy controls.

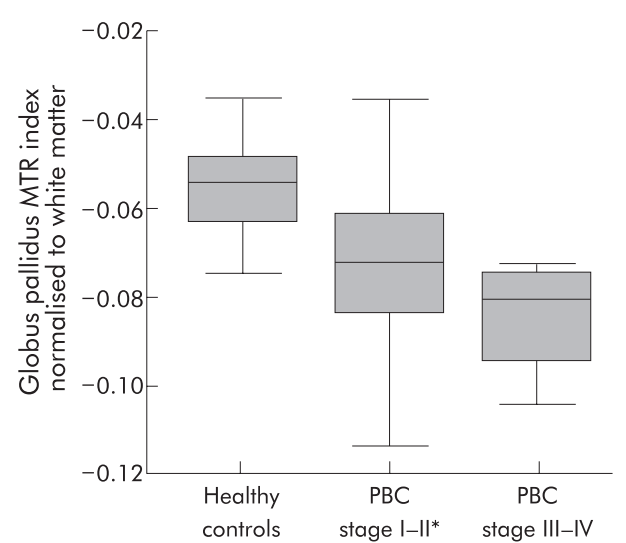

Figure 3 Box plot of the globus pallidus magnetisation transfer ratio (MTR) index normalised to white matter in the three study groups. Patients with stage I-II primary biliary cirrhosis (PBC) had a significantly reduced MTR index compared with healthy controls ( ${ }^{*} p=0.03$ ). The MTR index in stage III-IV patients was lower than that in stage I-II patients but this did not reach statistical significance. The boxes represent the interquartile range, which contain $50 \%$ of values, and the whiskers extend to the highest and lowest values. The median is represented by a line across the box. Differences were tested with the Kruskal-Wallis test $(p=0.01)$ and post hoc comparisons were made using the MannWhitney U test.
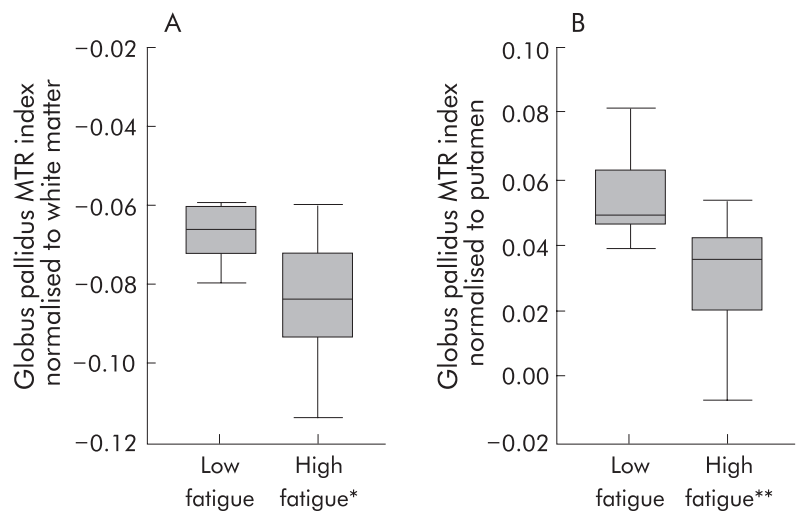

Figure 4 Box plots of the globus pallidus magnetisation transfer ratio (MTR) indices, normalised to white matter (A) and the putamen (B) in the high and low fatigue groups in stage I-II primary biliary cirrhosis patients. MTR indices were significantly reduced in the high fatigue group compared with the low fatigue group $\left({ }^{*} p=0.04,{ }^{* \star} p=0.02\right)$.

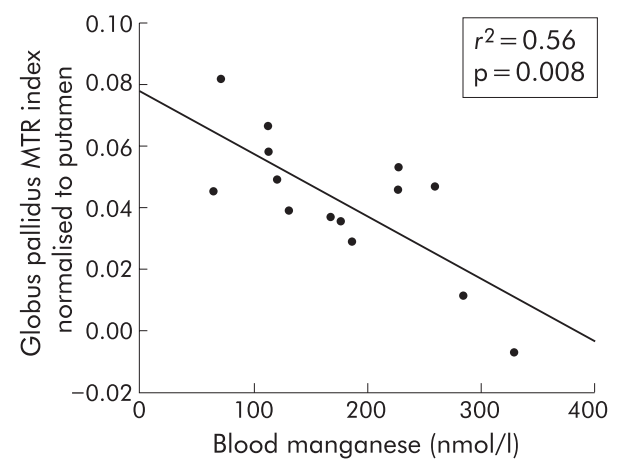

Figure 5 Correlation between the globus pallidus magnetisation transfer ratio (MTR) index normalised to the putamen, and blood manganese concentration. 
Table 2 Associations between the magnetic transfer ratio indices and blood manganese levels. Correlations were tested with Pearson's correlation coefficient

\begin{tabular}{llllll}
\hline $\begin{array}{l}\text { MTR index } \\
\text { normalised to WM }\end{array}$ & $\begin{array}{l}\text { Pearson } \\
\text { correlation } \\
\text { coefficient }\end{array}$ & $\mathbf{p}$ Value & $\begin{array}{l}\text { MTR index } \\
\text { normalised to PU }\end{array}$ & $\begin{array}{l}\text { Pearson } \\
\text { correlation } \\
\text { coefficient }\end{array}$ & $\mathrm{p}$ Value \\
\hline Globus pallidus & -0.54 & 0.196 & Globus pallidus & -0.75 & 0.008 \\
Head of caudate & -0.11 & 0.720 & Head of caudate & -0.53 & 0.156 \\
Thalamus & 0.09 & 0.761 & Thalamus & -0.25 & 0.393 \\
Putamen & 0.58 & 0.087 & White matter & -0.58 & 0.087 \\
\hline MTR, magnetisation transfer ratio; PU, putamen; WM, white matter. & & \\
\hline
\end{tabular}

correlation between the blood manganese concentration and the GP/PU index $(r=-0.75, \mathrm{p}=0.008)$ (fig 5$)$. There were no associations between the other ROI indices and the manganese level (table 2). In healthy controls, there was no association between age and MTR indices. However, in stage I-II PBC patients there was a significant correlation between patient age and the GP/PU MTR index $(r=0.55, \mathrm{p}=0.02)$.

\section{${ }^{1} \mathrm{H}$ Magnetic resonance spectroscopy}

There were no differences in the choline/creatine ratios between stage I-II PBC patients and healthy controls from the basal ganglia (mean choline/creatine (SD) stage I-II PBC 1.12 (0.15); healthy controls $1.15(0.19) ; \mathrm{p}=0.68)$ or from the white matter (stage I-II PBC $1.22(0.23)$; healthy controls $1.30(0.14) ; \mathrm{p}=0.38)$.

\section{DISCUSSION}

Fatigue is a multidimensional complaint that may be influenced by a number of factors, including physical symptoms and psychological determinants such as mood and personality. ${ }^{31}$ It is recognised as an important symptom in PBC and contributes significantly to impaired quality of life in this condition. ${ }^{5}$ The pathophysiological basis of fatigue in $\mathrm{PBC}$ is not known although experiments with bile duct resected rats have indicated that alterations in neurotransmission, ${ }^{11}$ the hypothalamic-pituitary axis, ${ }^{10}$ and/or brain sensitivity to cytokines ${ }^{12}$ may explain the observed reduction in locomotor activity in rats, the proposed correlate of human fatigue. There is a need for a physical marker of fatigue in humans with PBC as this symptom is highly subjective and its underlying cause is unclear.

In this study, we observed selectively reduced MTRs in the GP in patients with biopsy proven early (non-cirrhotic) liver disease and propose a pathogenic role for manganese in the development of fatigue in PBC. There have been numerous reports of increased signal intensity on standard $T_{1}$ weighted imaging in the GP in patients with established cirrhosis ${ }^{13}{ }^{15-19}$ which correlated with blood manganese levels. ${ }^{13} 1516$ Furthermore, two studies reported reduced GP MTRs in patients with cirrhosis which correlated with the severity of liver dysfunction. ${ }^{19}{ }^{32}$ It has been suggested that the reduction in MTR in patients with cirrhosis is due to low grade cerebral oedema as a consequence of hepatic encephalopathy. ${ }^{33}$ In this study, the main study group was confined to patients without cirrhosis, as assessed by liver biopsy. Our findings therefore are not due to hepatic encephalopathy. Furthermore, we observed no abnormality in cerebral ${ }^{1} \mathrm{H}$ MRS in stage I-II PBC patients. This finding emphasises the fact that the MTR results are not related to hepatic encephalopathy where the choline/creatine ratio is well documented to be reduced on ${ }^{1} \mathrm{H}$ MRS. ${ }^{26}{ }^{34}$ In addition, we have previously reported elevated choline/creatine MRS ratios in fatigued patients with hepatitis C and minimal (precirrhotic) liver disease. ${ }^{28}{ }^{29}$ The absence of such a finding in this study suggests that different mechanisms underlie the fatigue in PBC and hepatitis C infection.
There have been previous reports of increased GP signal intensity on standard MRI in patients with non-cirrhotic liver disease. ${ }^{35}{ }^{36}$ One study reported basal ganglia hyperintensity in patients with longstanding portal vein thrombosis, which correlated with serum manganese levels. ${ }^{35}$ Skehan et al reported GP hyperintensity on $\mathrm{T}_{1}$ weighted imaging in patients with chronic liver disease, which was more marked in patients with cholestatic disease (PBC and primary sclerosing cholangitis) than autoimmune hepatitis. ${ }^{36}$ They postulated that an unidentified metabolic process, unrelated to cirrhosis, hepatic encephalopathy, or portosystemic shunting, was the cause of their findings.

Reduced MTRs may result from pathologies that alter the structural integrity and the relative macromolecular water composition of brain parenchyma, such as multiple sclerosis plaques $^{37}$ or hepatic encephalopathy, ${ }^{33}$ or from deposition of paramagnetic substances. ${ }^{32}$ Magnetic resonance experiments with manganese chloride phantoms have demonstrated a strong inverse linear relationship between manganese concentration and MTR. ${ }^{32}$ This property has been exploited for the use of paramagnetic contrast agents such as gadolinium. In this study, there was a strong inverse correlation between whole blood manganese concentration and GP MTR indices, consistent with manganese deposition in the GP as a consequence of high blood levels due to impaired biliary excretion. This is supported by similar MR data in patients with hypermanganesaemia due to occupational exposure, ${ }^{20}$ total parenteral nutrition, ${ }^{22}$ and cirrhosis. ${ }^{15}{ }^{16}$ Furthermore, in one study of four cirrhotic patients, pre-mortem pallidal signal hyperintensity on $\mathrm{T}_{1}$ weighted imaging was associated with high post-mortem pallidal manganese concentrations. ${ }^{38}$ It is unclear whether MTRs change with age in the normal brain, with two recent studies finding no relationship ${ }^{39} 40$ and a third study reporting reductions after the age of 40 years. ${ }^{41}$ We found no association between age and MTR indices in healthy controls but we did find an association in stage I-II patients. A possible explanation for this is that, over time, greater quantities of manganese accumulate in the GP in individuals with a predisposition, such as chronic liver disease. The healthy controls in this study were significantly younger than the patients. This is a limitation of the study but in our view it is unlikely to be an important factor as any age related effect is likely to be very small.

Patients with high levels of fatigue had significantly lower pallidal MTR indices than those with less fatigue. In addition, there appeared to be an association between fatigue and blood manganese levels. The extrapyramidal and psychiatric signs and symptoms that are associated with manganism, occurring as a result of exposure to toxic levels of manganese, are generally more serious and florid than mere fatigue ${ }^{42}$ and were not seen in our patients. However, the early stages of low level exposure may be characterised by asthenia, anorexia, apathy, hypersomnia, weariness, and irritability. ${ }^{43} 44$ One might postulate that in PBC, elevation in brain manganese is much smaller and only manifests clinically 
with milder symptoms in individuals who are particularly sensitive.

Manganese is an abundant metal in the environment and is an essential element in humans, functioning as an enzyme cofactor and constituent of metalloenzymes. ${ }^{45}$ Its absorption is predominantly through the gastrointestinal tract where it is actively transported by the cationic transporter NRAMP II. ${ }^{46}$ In plasma, $80 \%$ of manganese is bound to $\beta_{1}$ globulin and albumin but a smaller fraction is bound to transferrin. The absorption and distribution of manganese are competitively inhibited by other cations, particularly iron. ${ }^{47}$ The major route of excretion is biliary and this serves as the main homeostatic mechanism. Elevated blood manganese concentrations occur in cirrhosis ${ }^{15}{ }^{16}$ and have also been reported in chronic hepatitis. ${ }^{48}$ Brain concentrations of manganese are relatively low and tightly regulated. Manganese is transported across the blood-brain barrier by a combination of facilitated diffusion, active transport of the non-protein bound cation, and transferrin dependent endocytosis in cerebral capillaries ${ }^{49}$ The GP, thalamic nuclei, and substantia nigra contain the highest manganese levels but do not correspond to regions with the highest transferrin receptor density. Rather, regions of high manganese concentration are efferent from regions rich in transferrin receptor density, suggesting that axonal transport of manganese may occur. ${ }^{50}$

The basal ganglia have multiple projections and participate in a number of parallel processes, including cognitive and emotional functions in addition to motor tasks. ${ }^{51}$ It has been suggested that any pathology that disrupts the pallidothalamo-cortical loop may result in reduced motivation, which may in turn be interpreted by an individual as centraltype fatigue. ${ }^{52}$ Indeed, fatigue is extremely common in Parkinson's disease and may antedate the development of motor symptoms. ${ }^{53}$ Patients with bilateral lesions of the basal ganglia, especially the GP, appear apathetic and demotivated in the absence of any motor symptoms. ${ }^{54}$ Although this is an attractive explanation of the neural basis of fatigue, it remains hypothetical in the current context. It is also possible that manganese deposition in brain structures may result in loss of neuronal and/or glial function leading to alterations in neurotransmission and intracerebral cytokine release. ${ }^{55}$ In this way, a link between our observations and the existing animal data, derived from bile duct resected rats, could be postulated.

The variation in the reported prevalence of fatigue in $\mathrm{PBC}$ may be due in part to the methods employed to measure fatigue. However, dietary manganese intake and other factors, including genetic factors, which determine absorption and distribution of manganese may explain why only a proportion of $\mathrm{PBC}$ patients experience fatigue. Indeed, iron deficiency may contribute to greater blood and tissue manganese levels, although in this study no relationship between blood manganese and iron was seen.

In summary, we observed reductions in the MTR in the GP in patients with stage I-II PBC which were strongly correlated with blood manganese levels. This preliminary observation together with other published data suggests that manganese deposition may occur in the GP in PBC in the absence of marked hepatic fibrosis or cirrhosis. The absence of an abnormality in cerebral MRS in these patients suggests that the reduced MTR is not a consequence of hepatic encephalopathy, which itself would not be expected in patients without cirrhosis. The association between fatigue severity and the pallidal MTR raises the possibility that brain manganese accumulation may be an important mechanism in the genesis of fatigue in patients with PBC. Our conclusions are limited by the size of this study. Further studies are warranted to investigate manganese homeostasis in a larger number of patients with $\mathrm{PBC}$ and to determine whether MRT abnormalities resolve in parallel with fatigue after liver transplantation. In addition, the possibility of ameliorating fatigue by dietary manganese restriction or iron supplementation requires further study.

\section{ACKNOWLEDGEMENTS}

The work was partly supported by Phillips Medical Systems International (Cleveland, Ohio, USA), the UK Department of Health, and the British Medical Research Council. Dr D Forton was supported by a grant from the European Association for the Study of the Liver. We are also grateful to LiverNorth, the patient support group based in Newcastle, for supporting this study.

\section{Authors' affiliations}

D M Forton, S D Taylor-Robinson, Liver Unit, Faculty of Medicine, St Mary's Hospital Campus, Imperial College London, London, UK, and Robert Steiner MR Unit, MRC Clinical Sciences Centre, Hammersmith Hospital Campus, Imperial College London, London, UK

H C Thomas, Liver Unit, Faculty of Medicine, St Mary's Hospital Campus, Imperial College London, London, UK

M Prince, J Goldblatt, M Bassendine, D E J Jones, Centre for Liver Research, University of Newcastle, Newcastle, UK

N Patel, A Oatridge, G Hamilton, J M Allsop, J V Hajnal, Robert Steiner MR Unit, MRC Clinical Sciences Centre, Hammersmith Hospital Campus, Imperial College London, London, UK

\section{REFERENCES}

1 Neuberger J. Primary biliary cirrhosis. Lancet 1997;350:875-9.

2 Prince MI, Jones DE. Primary biliary cirrhosis: new perspectives in diagnosis and treatment. Postgrad Med J 2000;76:199-206.

3 Witt-Sullivan $\mathrm{H}$, Heathcote $\mathrm{J}$, Cauch $\mathrm{K}$, et al. The demography of primary biliary cirrhosis in Ontario, Canada. Hepatology 1990;12:98-105.

4 Cauch-Dudek K, Abbey S, Stewart DE, et al. Fatigue in primary biliary cirrhosis. Gut 1998;43:705-10.

5 Huet PM, Deslauriers J, Tran A, et al. Impact of fatigue on the quality of life of patients with primary biliary cirrhosis. Am J Gastroenterol 2000;95:760-7.

6 Goldblatt J, Taylor PJ, Lipman T, et al. The true impact of fatigue in primary biliary cirrhosis: a population study. Gastroenterology 2002;122:1235-41.

7 Prince MI, James OF, Holland NP, et al. Validation of a fatigue impact score in primary biliary cirrhosis: towards a standard for clinical and trial use. J Hepatol 2000;32:368-73.

8 Goldblatt J, James OF, Jones DE. Grip strength and subjective fatigue in patients with primary biliary cirrhosis. JAMA 2001;285:2196-7.

9 Jalan R, Gibson H, Lombard MG. Patients with PBC have central but no peripheral fatigue. Hepatology 1996;24:A612.

10 Burak KW, Le T, Swain MG. Increased sensitivity to the locomotor-activating effects of corticotropin-releasing hormone in cholestatic rats. Gastroenterology 2002; 122:681-8.

11 Swain MG, Maric M. Improvement in cholestasis-associated fatigue with a serotonin receptor agonist using a novel rat model of fatigue assessment. Hepatology 1997;25:291-4.

12 Swain MG, Beck P, Rioux K, et al. Augmented interleukin-1 beta-induced depression of locomotor activity in cholestatic rats. Hepatology 1998;28:1561-5.

13 Krieger D, Krieger S, Jansen $O$, et al. Manganese and chronic hepatic encephalopathy. Lancet 1995;346:270-4.

14 Rose C, Butterworth RF, Zayed J, et al. Manganese deposition in basal ganglia structures results from both portal-systemic shunting and liver dysfunction. Gastroenterology 1999;1 17:640-4.

15 Hauser RA, Zesiewicz TA, Martinez C, et al. Blood manganese correlates with brain magnetic resonance imaging changes in patients with liver disease. Can J Neurol Sci 1996;23:95-8.

16 Spahr L, Butterworth RF, Fontaine S, et al. Increased blood manganese in cirrhotic patients: relationship to pallidal magnetic resonance signal hyperintensity and neurological symptoms. Hepatology 1996;24:11 16-20.

17 Zeneroli ML, Cioni G, Crisi G, et al. Globus pallidus alterations and brain atrophy in liver cirrhosis patients with encephalopathy: an MR imaging study. Magn Reson Imaging 1991;9:295-302.

18 Pujol J, Kulisevsky J, Moreno A, et al. Neurospectroscopic alterations and globus pallidus hyperintensity as related magnetic resonance markers of reversible hepatic encephalopathy. Neurology 1996;47:1526-30.

19 Taylor-Robinson SD, Oatridge A, Hajnal JV, et al. MR imaging of the basal ganglia in chronic liver disease: correlation of $\mathrm{T} 1$-weighted and magnetisation transfer contrast measurements with liver dysfunction and neuropsychiatric status. Metab Brain Dis 1995; 10:175-88.

20 Dietz MC, Ihrig A, Wrazidlo W, et al. Results of magnetic resonance imaging in long-term manganese dioxide-exposed workers. Environ Res 2001:85:37-40

21 Ikeda S, Yamaguchi Y, Sera Y, et al. Manganese deposition in the globus pallidus in patients with biliary atresia. Transplantation 2000;69:2339-43.

22 Mirowitz SA, Westrich TJ. Basal ganglial signal intensity alterations: reversal after discontinuation of parenteral manganese administration. Radiology 1992;185:535-6. 
23 Hajnal JV, Baudouin CJ, Oatridge A, et al. Design and implementation of magnetization transfer pulse sequences for clinical use. J Comput Assist Tomogr 1992;16:7-18.

24 Taylor-Robinson SD, Sargentoni J, Marcus CD, et al. Regional variations in cerebral proton spectroscopy in patients with chronic hepatic encephalopathy. Metab Brain Dis 1994;9:347-59.

25 Taylor-Robinson SD, Buckley C, Changani KK, et al. Cerebral proton and phosphorus-31 magnetic resonance spectroscopy in patients with subclinical hepatic encephalopathy. Liver 1999;19:389-98.

26 Taylor-Robinson SD, Sargentoni J, Oatridge A, et al. MR imaging and spectroscopy of the basal ganglia in chronic liver disease: correlation of $T_{1}$ weighted contrast measurements with abnormalities in proton and phosphorus-31 MR spectra. Metab Brain Dis 1996;11:249-68.

27 Ross BD, Jacobson S, Villamil F, et al. Subclinical hepatic encephalopathy: proton MR spectroscopic abnormalities. Radiology 1994;193:457-63.

28 Forton DM, Allsop JM, Main J, et al. Evidence for a cerebral effect of the hepatitis C virus. Lancet 2001;358:38-9.

29 Forton DM, Thomas HC, Murphy CA, et al. Hepatitis C and cognitive impairment in a cohort of patients with mild liver disease. Hepatology 2002;35:433-9.

30 Miller BL. A review of chemical issues in 1H NMR spectroscopy: N-acetyl-Laspartate, creatine and choline. NMR Biomed 1991;4:47-52.

31 Wessely S. Chronic fatigue: symptom and syndrome. Ann Intern Med $2001 ; 134: 838-43$

32 Iwasa M, Kinosada Y, Nakatsuka A, et al. Magnetization transfer contrast of various regions of the brain in liver cirrhosis. AJNR Am J Neuroradiol 1999;20:652-4.

33 Cordoba J, Alonso J, Rovira A, et al. The development of low-grade cerebral edema in cirrhosis is supported by the evolution of ${ }^{1} \mathrm{H}$-magnetic resonance abnormalities after liver transplantation. J Hepatol 2001;35:598-604.

34 Bluml S, Zuckerman E, Tan J, et al. Proton-decoupled ${ }^{31} \mathrm{P}$ magnetic resonance spectroscopy reveals osmotic and metabolic disturbances in human hepatic encephalopathy. J Neurochem 1998;71:1564-76.

35 Nolte W, Wilffang J, Schindler CG, et al. Bright basal ganglia in T1-weighted magnetic resonance images are frequent in patients with portal vein thrombosis without liver cirrhosis and not suggestive of hepatic encephalopathy. J Hepatol 1998;29:443-9.

36 Skehan S, Norris S, Hegarty J, et al. Brain MRI changes in chronic liver disease. Eur Radiol 1997;7:905-9.

37 van Buchem MA, Tofts PS. Magnetization transfer imaging. Neuroimaging Clin N Am 2000;10:771-88.

38 Maeda $H$, Sato $M$, Yoshikawa A, et al. Brain MR imaging in patients with hepatic cirrhosis: relationship between high intensity signal in basal ganglia on $\mathrm{T}_{1}$-weighted images and elemental concentrations in brain. Neuroradiology 1997;39:546-50

39 Mehta RC, Pike GB, Enzmann DR. Magnetization transfer MR of the normal adult brain. AJNR Am J Neuroradiol 1995;16:2085-91.

40 Rovaris $M$, lannucci $G$, Cercignani $M$, et al. Age-related changes in conventional, magnetization transfer, and diffusion-tensor MR imaging findings: study with whole-brain tissue histogram analysis. Radiology 2003;227:731-8.

$41 \mathrm{Ge} Y$, Grossman RI, Babb JS, et al. Age-related total gray matter and white matter changes in normal adult brain. Part ll: quantitative magnetization transfer ratio histogram analysis. AJNR Am J Neuroradiol 2002;23:1334-41.

42 Lee JW. Manganese intoxication. Arch Neurol 2000;57:597-9.

43 Bowler RM, Mergler D, Sassine MP, et al. Neuropsychiatric effects of manganese on mood. Neurotoxicology 1999;20:367-78.

44 Roels H, Lauwerys R, Buchet JP, et al. Epidemiological survey among workers exposed to manganese: effects on lung, central nervous system, and some biological indices. Am J Ind Med 1987;11:307-27.

45 Wedler FC. Biological significance of manganese in mammalian systems. Prog Med Chem 1993;30:89-133.

46 Conrad ME, Umbreit JN, Moore EG, et al. Separate pathways for cellular uptake of ferric and ferrous iron. Am J Physiol Gastrointest Liver Physiol 2000;279:G767-74.

47 Malecki EA, Devenyi AG, Beard JL, et al. Existing and emerging mechanisms for transport of iron and manganese to the brain. J Neurosci Res 1999;56:113-22.

48 Versieck J, Barbier F, Speecke A, et al. Manganese, copper, and zinc concentrations in serum and packed blood cells during acute hepatitis, chronic hepatitis, and posthepatitic cirrhosis. Clin Chem 1974;20:1141-5.

49 Aschner M, Vrana KE, Zheng W. Manganese uptake and distribution in the central nervous system (CNS). Neurotoxicology 1999;20:173-80.

50 Sloot WN, Gramsbergen JB. Axonal transport of manganese and its relevance to selective neurotoxicity in the rat basal ganglia. Brain Res 1994;657: 124-32

51 Middleton FA, Strick PL. Basal ganglia output and cognition: evidence from anatomical, behavioral, and clinical studies. Brain Cogn 2000;42:183-200.

52 Chaudhuri A, Behan PO. Fatigue and basal ganglia. J Neurol Sci 2000;179:34-42.

53 Friedman J, Friedman H. Fatigue in Parkinson's disease. Neurology 1993;43:2016-18.

54 Laplane D, Baulac M, Widlocher D, et al. Pure psychic akinesia with bilateral lesions of basal ganglia. J Neurol Neurosurg Psychiatry 1984:47:377-85

55 Chen CJ, Liao SL. Oxidative stress involves in astrocytic alterations induced by manganese. Exp Neurol 2002;175:216-25. 